\title{
PERCEPCIÓN DE MUJERES VENEZOLANAS SOBRE LOS SERVICIOS DE SALUD SEXUAL Y REPRODUCTIVA EN LIMA, PERÚ
}

\author{
Rebecca Irons (1) 1 a \\ ${ }^{1}$ University College London, Londres, Reino Unido. \\ a Antropóloga, doctora en Antropología de la Medicina.
}

\section{RESUMEN}

Objetivos: Conocer la percepción de mujeres venezolanas sobre los servicios de salud sexual y reproductiva (SSR) en Lima, Perú. Materiales y métodos: Estudio con enfoque metodológico cualitativo; se entrevistó a 50 mujeres migrantes en Lima y tres obstetras que brindan atención en SSR por el Fondo de Población de las Naciones Unidas, la información fue recolectada de julio a diciembre de 2019. Se analizó la prestación de servicios de SSR en Lima a través de la teoría de la "gobernanza reproductiva». Resultados: Las mujeres migrantes buscan información en salud reproductiva y anticonceptivos en farmacéuticos y farmacias. Existe un déficit de información sobre la atención gratuita de los SSR, preocupación por la falta de seguro de salud, por las barreras de costos y por su estado migratorio. Conclusiones: La «gobernanza reproductiva» podría ser empleada por los farmacéuticos para apoyar a las mujeres migrantes y para informar sobre los servicios públicos de SSR disponibles en Lima.

Palabras clave: Migrantes; Salud sexual; Venezuela; Perú; Anticonceptivos (fuente: DeCS BIREME)

\section{VENEZUELAN WOMEN'S PERCEPTION OF SEXUAL AND REPRODUCTIVE HEALTH SERVICES IN LIMA, PERU}

\begin{abstract}
Objectives: To know the perception of Venezuelan women about sexual and reproductive health (SRH) services in Lima, Peru. Materials and methods: This study had a qualitative methodological approach; 50 migrant women in Lima and three obstetricians who provide SRH care by the United Nations Population Fund were interviewed, collected from July to December 2019. The SRH service in Lima was analyzed using the theory of "reproductive governance". Results: Migrant women seek reproductive health and contraceptive information from pharmacists and pharmacies. There is an information deficit on free SRH care, concerns about lack of health insurance, cost barriers, and their migration status. Conclusions: "Reproductive governance" could be employed by pharmacists to support migrant women and to inform about public SRH services available in Lima.
\end{abstract}

Keywords: Migrants; Sexual health; Venezuela; Peru; Contraception (source: MeSH NLM)

Citar como: Irons R. Percepción de mujeres venezolanas sobre los servicios de salud sexual y reproductiva en Lima, Perú. Rev Peru Med Exp Salud Publica. 2021;38(2):248-53. doi: https://doi. org/10.17843/rpmesp.2021.382.6217

Correspondencia: Rebecca Irons; University College London, 14 Taviton Street, Bloomsbury, WC1H 0BW, Reino Unido; rebecca.irons@ucl.ac.uk

Recibido: 30/12/2020 Aprobado: 26/05/2021 En línea: 30/06/2021

\section{INTRODUCCIÓN}

La última década en Venezuela ha sido marcada por la inflación y la inestabilidad socioeconómica que ha afectado la atención en salud en el país, lo que ha generado que la búsqueda de atención médica sea una de las principales necesidades y razones para la migración de los venezolanos ${ }^{(1)}$. El Perú recibió aproximadamente 800000 migrantes hasta 2019, siendo el segundo mayor receptor después de Colombia ${ }^{(1)}$. Perú es el principal país anfitrión para aquellos que buscan el estatus de refugiado, y ha otorgado un permiso temporal de permanencia (PTP) a casi la mitad de los venezolanos legalmente registrados que han ingresado al país ${ }^{(1)}$. Estas condiciones favorables se extienden a la salud en Perú en cierta medida, ya que los migrantes tienen permiso legal para acceder a ciertas especialidades médicas que están disponibles a nivel nacional, independientemente de su estatus migratorio o nacionalidad. 
Entre estos servicios existen los de salud sexual y reproductiva (SSR), como la orientación de planificación familiar y métodos anticonceptivos, y atención del embarazo y del menor de cinco años que se ofrecen de forma gratuita en los centros de salud del Ministerio de Salud del Perú (MINSA). Para utilizar los servicios de salud públicos más allá de estas especialidades, uno debe tener el Seguro Integral de Salud (SIS) ${ }^{(2)}$, que no está disponible para la mayoría de la población migrante ya que requiere residencia permanente más allá del PTP.

Entre las diversas necesidades de salud de los migrantes, la atención reproductiva y la planificación familiar son un área importante que debe priorizarse ${ }^{(3)}$. Casi la mitad de los migrantes que llegan a Perú son mujeres $(47,7 \%)$, que pueden enfrentar meses de precariedad y exposición a la vulnerabilidad y la violencia ${ }^{(4)}$. De hecho, se ha destacado que el género es una cuestión clave en relación con la salud de los migrantes que debe abordarse con urgencia en los esfuerzos de respuesta sanitaria mundial ${ }^{(5,6)}$. El embarazo no planificado puede afectar especialmente a las mujeres migrantes, tanto durante el período de viaje como a su llegada, donde pueden enfrentar inseguridad económica y problemas de salud.

En 2018, el Instituto Nacional de Estadística e Informática (INEI) de Perú realizó un censo de la población venezolana que vive en Perú, que nos proporciona algunos datos cuantitativos preliminares sobre las condiciones de salud y los servicios utilizados por los migrantes en años recientes ${ }^{(4)}$. Es poca la información obtenida sobre su experiencia y conocimiento respecto a cómo acceder a los servicios de salud, por ese motivo, el interés en investigar la situación sanitaria de los migrantes es cada vez mayor.

El presente estudio buscó conocer la percepción de mujeres venezolanas sobre los servicios de SSR en Lima, Perú. Se argumentará que la situación actual con los servicios de SSR para las mujeres migrantes venezolanas en Lima debe analizarse utilizando el marco teórico de la gobernanza reproductiva ${ }^{(7)}$, que son los mecanismos a través de los cuales algunos actores como el estado, la sociedad civil y las organizaciones no gubernamentales (ONG) dan forma e intervienen en las decisiones reproductivas de las mujeres. Asimismo, se extenderá el marco teórico para proponer que la gobernanza reproductiva no se aplique solo cuando los actores utilizan instrumentos legislativos, incentivos económicos, principios morales o éticos, o coerción directa para orientar y controlar el comportamiento y las prácticas reproductivas, sino también cuando un actor influye en las decisiones reproductivas de las mujeres.

\section{MATERIALES Y MÉTODOS}

El diseño del estudio utilizó una metodología cualitativa de investigación, centrada en entrevistas en profundidad en

\section{MENSAJES CLAVE}

Motivación para realizar el estudio: Con un número cada vez mayor de migrantes venezolanas que llegan al Perú, es importante cubrir sus necesidades de salud e información sobre los servicios de salud sexual y reproductiva (SSR). Sin embargo, existe una falta de estudios cualitativos que aborden este problema.

Principales hallazgos: Los principales hallazgos sugieren que las migrantes pagan por los SSR en las farmacias, a pesar de que estos servicios son gratuitos a través del Ministerio de Salud.

Implicancias: Se necesita informar a las migrantes sobre las oportunidades gratuitas de atención médica disponibles, y se recomienda que se brinde información en farmacias, donde la mayoría de las migrantes van a buscarlo.

Lima y en los SRR del Fondo de Población de las Naciones Unidas (UNFPA). El reporte del estudio siguió la guía COREQ ${ }^{(8)}$. La investigación se basa en teorías antropológicas y sociológicas de la salud y considera los objetivos y el contexto social en su totalidad ${ }^{(9,10)}$.

\section{Procedimiento}

\section{Selección de las participantes}

Se seleccionaron a las participantes utilizando muestreo por conveniencia a través de contactos del UNFPA y la Unión Venezolana en Lima. Entre julio a diciembre de 2019, se entrevistó personalmente a 50 mujeres venezolanas que viven en Perú (Tabla 1) y se realizaron entrevistas no estructuradas a tres obstetras mujeres del UNFPA que brindan servicios de SSR. No se excluyeron participantes y los datos se recolectaron en los lugares acordados a conveniencia de la persona entrevistada (por ejemplo, su lugar de trabajo).

\section{Recopilación de la información}

Todas las entrevistas se completaron sin intervención externa. El rango de edad de la muestra era entre 18 y 49 años. La entrevista siguió un formato estructurado con indicaciones de la investigadora, para explicar las preguntas con detenimiento o para analizar la situación cuando fuera necesario. Las entrevistas se grabaron en audio cuando se obtuvo el permiso del participante. Tuvieron una duración entre 15 a 30 minutos y se realizaron en una sola sesión. Las entrevistas con las mujeres participantes no alcanzaron la saturación teórica. Las transcripciones no fueron entregadas a las participantes, porque ninguna lo pidió.

Todas las entrevistas fueron completadas por la autora del estudio. La investigadora posee un doctorado en Antropología Médica de la University College London (UCL) y ha realizado estudios etnográficos con poblaciones rurales y 
Tabla 1. Información demográfica de las participantes

\begin{tabular}{|c|c|}
\hline Característica & $\mathbf{n}$ \\
\hline \multicolumn{2}{|l|}{ Edad (años) } \\
\hline $18-21$ & 4 \\
\hline $22-29$ & 8 \\
\hline $30-45$ & 24 \\
\hline $45-59$ & 14 \\
\hline \multicolumn{2}{|l|}{ Lugar de origen } \\
\hline Anzoátegui & 3 \\
\hline Aragua & 4 \\
\hline Bolívar & 3 \\
\hline Carabobo & 1 \\
\hline Caracas & 18 \\
\hline Cojedes & 1 \\
\hline Falcón & 4 \\
\hline Lara & 5 \\
\hline Táchira & 1 \\
\hline Trujillo & 1 \\
\hline Yaracuy & 7 \\
\hline Zulia & 2 \\
\hline \multicolumn{2}{|l|}{ Distrito de residencia } \\
\hline Ate & 2 \\
\hline Callao & 1 \\
\hline Cercado & 1 \\
\hline Chorrillos & 7 \\
\hline La Victoria & 2 \\
\hline Los Olivos & 11 \\
\hline Pachacámac & 3 \\
\hline Rímac & 2 \\
\hline Santa Anita & 3 \\
\hline San Juan de Lurigancho & 11 \\
\hline San Martín de Porres & 1 \\
\hline Surco & 4 \\
\hline Villa el Salvador & 2 \\
\hline
\end{tabular}

urbanas en el Perú. La investigadora y las participantes del estudio se conocieron el día de la entrevista. Se informó a las participantes sobre los objetivos del estudio antes de que estos consintieran su participación. Asimismo, se informó a la institución de la investigadora (UCL) y a la institución colaboradora (UNFPA) sobre la realización del estudio.

\section{Análisis estadístico}

Los datos fueron codificados, sin la utilización de un árbol de codificación. Los temas se derivaron una vez recopilados todos los datos y aquellos que se presentaron con mayor frecuencia fueron seleccionados para ser analizados. Las transcripciones de las entrevistas fueron analizadas detalladamente para seleccionar los datos que representaron mejor los temas.
Se usó el software de reconocimiento de voz Dragon versión 6.0.0 (www.nuance.com) para ayudar con la transcripción de la entrevista. Las identidades de los participantes fueron codificadas por edad y ciudad de origen, pero no se las numeró. Hubo coherencia entre los datos presentados y los hallazgos.

\section{Aspectos éticos}

El estudio fue aprobado por el comité de ética de la UCL con el código: 10285/002.

\section{RESULTADOS}

\section{Déficit de información}

Para obtener información sobre el acceso a los SSR disponibles, los migrantes deberían haberse puesto en contacto con representantes del MINSA u otras ONG que operan en el país y en las fronteras (como UNFPA, Agencia de la Naciones Unidas para los Refugiados, Cruz Roja). La ruta migratoria más común de Venezuela a Perú es por tierra a través de Colombia y Ecuador, y la mayoría ingresa al país a través de Tumbes. Los que llegan a Tumbes pasarán por los Centros Binacionales de Atención en Frontera (CEBAF). Aquí los migrantes pueden solicitar PTP o asilo, como lo hicieron 110000 en 2019, además de recibir atención médica de organizaciones humanitarias, incluida la atención y orientación sobre SSR a través del UNFPA. Un total de 43 participantes en este estudio llegaron por tierra, pasando por Tumbes.

Es importante destacar que pasar por el CEBAF supone la entrada legal al país, lo que puede no haber sido necesariamente el caso. Además, no todos los migrantes podrían haber sido dirigidos hacia los centros médicos de los CEBAF y, por lo tanto, es posible que no hayan recibido ninguna información sobre los servicios de salud en el país. En Perú, 47 participantes fueron directamente a Lima, 2 vivían en Piura y 1 en Jauja antes de mudarse a la capital, lo que sugiere que, si se pierde la oportunidad de contactarlas en el CEBAF, entonces es más difícil localizarlas en Lima. Solo una participante mencionó que había asistido a los servicios de salud de los CEBAF, y esta fue su única atención médica en Perú. No hubo un conocimiento adecuado ni información correcta sobre el acceso a los servicios de SSR en Lima entre las mujeres migrantes. Solo 12 mujeres sabían que se podía visitar un centro de salud de MINSA para obtener servicios de SSR gratuitos o de bajo costo.

Casi la mitad de las participantes expresaron su preferencia por los centros de salud del MINSA (24/50), un 32\% por farmacias $(16 / 50)$ y un $20 \%$ por clínicas privadas $(10 / 50)$. Cuando se les preguntó específicamente dónde buscarían anticonceptivos, solo ocho respondieron que en el MINSA, y las 42 restantes dijeron que visitarían una farmacia para comprarlos. 
Casi la mitad de las mujeres entrevistadas no sabían que pueden recibir anticonceptivos y servicios de planificación familiar de forma gratuita. Solo 4 participantes declararon explícitamente que sabían que podían recibir anticonceptivos de forma gratuita a través del MINSA, y en todos los casos habían descubierto esta información porque habían visitado un centro de salud por otra razón: dos cuando acompañaban a sus hijos para recibir atención médica, y dos por embarazo. En ambos casos de embarazo, las mujeres no sabían que podían recibir atención médica gratuita. Una (34, San Juan de Miraflores [SJM]) asistió a un ginecólogo privado durante los primeros 4 meses, porque el MINSA «no quería atenderla» a pesar de que su esposo era de nacionalidad peruana, y la otra (22, San Juan de Lurigancho [SJL]) pagó por servicios médicos privados durante los primeros 5 meses, y solo se enteró de la atención gratuita porque otros migrantes se lo contaron. Sin embargo, una vez que visitó el MINSA, dijo que era fácil obtener el SIS.

\section{Seguro Integral de Salud (SIS)}

Las mujeres tuvieron la percepción de que el SIS era un requisito necesario para acceder a todos los servicios. De las participantes entrevistadas, solo 4 tenían SIS y 2 de ellas lo tenían porque habían estado embarazadas. Sin embargo, no solo las mujeres migrantes pueden estar confundidas con este detalle, sino también algunos trabajadores de la salud. Por ejemplo, una entrevistada embarazada que tuvo que pagar por servicios médicos privados durante los primeros cuatro meses de embarazo tuvo obstáculos en el centro de salud del MINSA ya que el personal no sabía si brindarle atención materna sin SIS y cómo hacerlo.

El debate sobre los migrantes y el acceso al SIS también prevalece en la opinión pública, por ejemplo, los grafitis en un área de alta densidad de migrantes (SJL) dice: «SIS para peruanos» (Figura 1). Por lo tanto, las preocupaciones sobre la extensión del SIS a los migrantes, incluso por parte de algunos trabajadores de la salud, han prevalecido en los últimos años debido al contexto político y los temores de que el sistema pueda verse colapsado y revertir el acceso del ciudadano peruano a la atención médica gratuita.

\section{Costos}

Las mujeres migrantes estaban particularmente preocupadas por los costos asociados con la obtención de atención y anticonceptivos en los SSR. Dieciséis participantes de Lima no tenían trabajo, y seis eran vendedoras ambulantes. Dado que el $84 \%$ de los participantes creía que era necesario comprar anticonceptivos en una farmacia, les resultaba muy difícil comprarlos. Dejando de lado los bajos salarios, había una percepción de que la medicina y la atención médica en Perú eran costosos en general: «No voy a visitar al médico porque tienes que pagar por todo, muchas pruebas, todo es pagado»

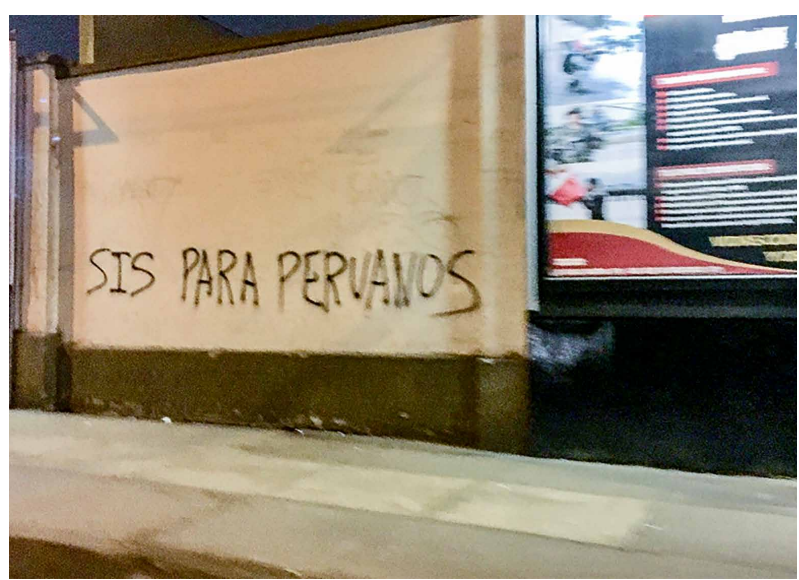

Fuente: fotografía tomada por la autora.

Figura 1. Grafiti «SIS para peruanos» (Lima, agosto de 2019).

(44, Surco); «Aquí [en el hospital] te hacen gastar dinero innecesariamente» (39, SJL). Una participante (25, Surquillo) comentó que existía una "gran diferencia» en comparación con Venezuela, donde todo era gratis bajo el sistema socialista. Sin embargo, también afirmó que «es por eso por lo que Venezuela se encuentra en esta situación, porque todo era gratis». Otra participante (30, Pachacámac) escucho que, si quedaba embarazada y necesitaba una cesárea, le costaría S/ 2000 (\$600).

Existían dos obstáculos principales en términos de percepción de costos. La primera es que, debido a que las mujeres estaban preocupadas por el costo de los servicios y tenían una escasez de información al respecto, no visitaron los establecimientos del MINSA y, por lo tanto, no se enteraron de que algunos servicios eran gratuitos. El segundo problema es que, incluso cuando los trabajadores de salud estaban en condiciones de informar a las mujeres migrantes sobre los servicios gratuitos, no lo hicieron. Esto está ejemplificado por las dos mujeres embarazadas que pasaron casi la mitad de su período de gestación pagando por la atención del embarazo cuando debería haber sido gratuita.

\section{Estado migratorio}

A partir de 2018, los migrantes debían tener pasaportes para ingresar al Perú, mientras que antes su cédula nacional era suficiente. Los migrantes que habían ingresado antes del cambio de ley y no tenían un pasaporte expresaron su preocupación acerca de si se requería o no un pasaporte para recibir anticonceptivos gratuitos.

Estos no fueron casos en los que el migrante se encontraba ilegalmente en el país, solo que a medida que las leyes de entrada cambiaban les preocupaba no tener la documentación apropiada y que sea una excusa para negarles el acceso a los servicios. Un total de 12 participantes sin SIS dijeron que fueron rechazadas en un centro de salud del 
MINSA porque no tenían la ciudadanía peruana. Un escenario importante que merece atención aquí fue presentado por una mujer que había dado a luz por cesárea en Lima (34, SJM). Cuando ingresó en el hospital para una cesárea programada, necesitaba una transfusión de sangre, sin embargo, le informaron que los donantes para transfusiones deben poseer la ciudadanía peruana.

\section{DISCUSIÓN}

Uno de los mayores problemas para las mujeres migrantes cuando se trata de acceder a los servicios de SSR en Lima es el notable déficit de información disponible para ellas. Si bien es posible recibir información al ingresar a Perú, muchas mujeres parecen no recibirla y no tienen otra oportunidad de abordar sus brechas de conocimiento en materia de salud. Debido a esto, se agravan otros factores que existen como barreras de acceso. Por ejemplo, las mujeres van a las farmacias para comprar anticonceptivos en lugar de recibirlos gratis y los consideran demasiado costosos, especialmente teniendo en cuenta su escaso salario. Entonces sienten frustración con el sistema de salud, ya que las mujeres sienten que deberían proveer anticonceptivos gratuitos, sin saber que son ofrecidos gratuitamente en los centros de salud del MINSA. Con más información, estos problemas podrían haberse evitado y las mujeres tendrían la oportunidad de obtener asesoría sobre planificación familiar de forma gratuita en los centros del MINSA.

Sin embargo, hay un lugar donde las mujeres acuden con frecuencia para recibir atención médica: la farmacia. El censo del INEI de 2018 encontró que el 52,5\% de las mujeres encuestadas preferían buscar atención médica en las farmacias, y el 19\% eligió los centros de salud del MINSA ${ }^{(4)}$. Así, los farmacéuticos pueden actuar como contactos clave tanto para el acceso a los anticonceptivos como para el asesoramiento y la orientación de planificación familiar. Esto sugiere «gobernanza reproductiva» ${ }^{(7)}$ por parte de las farmacias/farmacéuticos, y si se aborda adecuadamente, esto puede proporcionar un punto de entrada clave para la difusión de información adecuada a las mujeres migrantes. Una recomendación para abordar el déficit de información es capacitar a los farmacéuticos en áreas de alta densidad de migrantes para que orienten a las mujeres a que usen los servicios de planificación familiar de los centros de salud y no dependan únicamente de las compras en la farmacia. Asimismo, se pueden colocar carteles en las farmacias de Lima advirtiendo a las mujeres migrantes que tienen derecho a algunos servicios de forma gratuita en su centro local de salud MINSA y también brindar asesoramiento y orientación más allá de lo que se puede encontrar en una farmacia.

La venta ambulatoria es una actividad laboral realizada por el $46 \%$ de los venezolanos en Perú que genera pocos ingresos y expone a las mujeres al acoso sexual ${ }^{(11,12)}$. Por ello, el costo de los SSR fue considerado como una barrera de acceso. Esta creencia puede provenir de discursos nacionalistas en Venezuela sobre el estado socialista y la atención de la salud. Como Cooper ha argumentado ${ }^{(13)}$, el modelo del sistema de salud socialista desarrollado a partir de la revolución bolivariana no solo tuvo éxito en extender la atención médica a las personas marginadas, sino que funcionó en la construcción de una nación socialista que fue, sobre todo, inclusiva, independientemente de necesidad económica. Es importante destacar que los sistemas de salud socialistas pueden presentarse a sus naciones como únicos y causar un malentendido de que la medicina social no estará disponible en otros países, como Perú ${ }^{(14,15)}$. Cuando los migrantes comparan la atención médica venezolana con la de Perú, es probable que no se refieran a los últimos años; de hecho, las mujeres que hicieron esos comentarios comparativos particulares habrían sido adultas durante el apogeo político de Chávez, a diferencia de las migrantes más jóvenes que habrían llegado hasta la edad adulta durante un período de incertidumbre económica y política ${ }^{(15,16)}$. Cuando las personas no tienen mucha experiencia con el sistema de salud del país anfitrión, se sorprenden de que los medicamentos sean gratuitos en el país que los acoge.

Una última cuestión es el estado migratorio que rodea y cómo esto puede causar una barrera para que las mujeres accedan a los servicios de SSR. Esto no debería afectar su capacidad de recibir anticonceptivos gratuitos legalmente, sin embargo, esto puede ser percibido como un área gris incluso para aquellas que han ingresado al país con la documentación correcta, ya que pueden evitar interferencias legales hasta que tengan sus documentos en orden. Además, el sistema está en gran parte no digitalizado, lo que significa que no hay información electrónica para que un personal de salud del MINSA pueda discernir rápidamente el estado legal de un migrante e informarlo a las autoridades fronterizas. Si bien un sistema basado en papel es problemático dentro del MINSA y otros sistemas estatales, ya que reduce la eficiencia ${ }^{(17-19)}$ en este caso podría ayudar a los migrantes que puedan estar preocupados por una vigilancia estatal. Las recomendaciones anteriores que tienen como objetivo difundir más información a las mujeres migrantes sobre el sistema de salud peruano permitirían que más mujeres experimenten estos cambios, y que en la práctica su falta de documentos no sea una barrera para recibir servicios de SSR.

Dentro de las limitaciones del estudio se incluyen el impedimento para conocer profundamente a las migrantes que participaron ${ }^{(20)}$ y que no se alcanzó la saturación de datos. Además, entre los migrantes venezolanos en el Perú, existe una gran diversidad de niveles de ingresos y de preferencias de servicios de salud ${ }^{(4)}$, pero no fue posible recolectar ni analizar todas las diferencias en el presente estudio. 
En la actualidad, hay servicios de salud disponibles para las venezolanas en Lima, sin embargo, hay una serie de barreras que limitan el acceso y el conocimiento sobre estos. Hasta cierto punto, estos pueden abordarse a través de la difusión de información específica en sitios claves donde los migrantes realmente van en busca de información sobre SSR y productos farmacéuticos como son las farmacias. Esto sugiere que estos sitios están promulgando «gobernanza reproductiva» sobre las mujeres migrantes, y como se ha sugerido, si se gestiona adecuadamente, esto podría usarse como una buena oportunidad para informarles mejor sobre los servicios disponibles.

\section{REFERENCIAS BIBLIOGRÁFICAS}

1. United Nations High Commission on Refugees (UNHCR). Fact sheet: Peru June 2019 [Internet]. 2019 [citado el 9 de agosto de 2020]. Disponible en: https://www.acnur.org/5d046a174.pdf.

2. Irons R. Planning Quechua Families: Indigenous Subjectivities, Inequalities and Kinship under the Peruvian Family Planning Programme. Doctoral thesis, UCL (University College London). 2020. Disponible en: https://discovery.ucl.ac.uk/id/eprint/10097353/.

3. Albaladejo A. Contraceptive shortages mean Venezuela's people face a sexual health emergency. BMJ. 2018;360:k1197. doi:10.1136/bmj.k1197.

4. Instituto Nacional de Estadística e Informática. Condiciones de vida de la población Venezolana que reside en Perú. Lima; 2019.

5. Cousins S. A complex epidemic prevents Peru from reaching HIV goals. Lancet HIV. 2019,6(11):733-734. doi: 10.1016/S2352-3018(19)30351-0.

6. Orcutt M, Spiegel P, Kumar B, Abubakar I, Clark J, Horton R. Lancet Migration: global collaboration to advance migration health. Lancet. 2020;395(10221):317-9. doi: 10.1016/S0140-6736(20)30107-0.

7. Morgan LM, Roberts EFS. Reproductive governance in Latin America. Anthropol Med. 2012;19(2):241-54. doi: 10.1080/13648470.2012.675046.

8. Tong A, Sainsbury P, Craig J. Consolidated criteria for reporting qualitative research (COREQ): a 32-item checklist for interviews and focus groups. Int J Qual Health Care. 2007;19(6):349-57. doi: 10.1093/intqhc/mzm042.

9. do Prado ML, de Souza M de L, Carraro TE. Investigación cualitativa en enfermería: contexto y bases conceptuales. Washington, DC; 2008.

10. Guanipa PM. Objetivos y propósitos en la investigación: una dialéctica en las ciencias sociales [Internet]. Gestiopolis; 2008 [citado el 9 de junio de 2020]. Disponible en: https://www.gestiopolis.com/objetivos-propositos-investigacion-dialectica-ciencias-sociales/.

11. Instituto de Opinión Pública, Pontificia Universidad Católica del Perú. Cambios en las Actitudes hacia los Inmigrantes Venezolanos en Lima-Cal-
Agradecimientos: A Walter Mendoza, del Fondo de Población de las Naciones Unidas (UNFPA) en Lima, Perú, por su apoyo durante este proyecto, y a Wellcome Trust quien financió el proyecto.

Contribuciones de los autores: RI concibió el estudio, redactó el manuscrito, aprobó la versión final que se publicará, y asume la responsabilidad frente a todos los aspectos del manuscrito.

Financiamiento: El proyecto fue financiado por Wellcome Trust, número de subvención: 218312 / Z / 19 / Z.

Conflictos de interés: La autora declara no tener conflictos de interés.

lao 2018-2019 [Internet]. Lima: Pontificia Universidad Católica del Perú; 2020 [citado el 9 de junio de 2020]. Disponible en: http://repositorio.pucp. edu.pe/index/handle/123456789/169459.

12. Freier LF. Understanding the Venezuelan Displacement Crisis [Internet]. E-International Relations; 2018 [citado el 9 de junio de 2020]. Disponible en: https://www.e-ir.info/2018/06/28/understanding-the-venezuelan-displacement-crises/.

13. Cooper A. State of health: pleasure and politics in Venezuelan health care under Chávez. University of California Press; 2019.

14. Andaya E. The gift of health: Socialist medical practice and shifting material and moral economies in post-Soviet Cuba. Med Anthropol Q. 2009;23(4):357-74. doi: 10.1111/j.1548-1387.2009.01068.x.

15. Brotherton PS. "We have to think like capitalists but continue being socialists": Medicalized subjectivities, emergent capital, and socialist entrepreneurs in post-Soviet Cuba. Am Ethnol. 2008;35(2):259-74. doi:. 10.1111/j.2008.1548-1425.00033.x.

16. Gallegos R. Crude nation: How oil riches ruined Venezuela. first edit. Books P. Lincon: University of Nebraska Press; 2016. 1-226 p. Disponible en: http://digitalcommons.unl.edu/unpresssampleshttp://digitalcommons.unl.edu/unpresssamples/326.

17. Rodríguez F. Venezuela's Revolution in Decline: Beware the Wounded Tiger. World Policy Journal, 2008,25(1):45-58.

18. Yrivarren Espinoza J. Gobierno Electrónico: Análisis de los conceptos de tecnología, comodidad y democracia. Universidad Peruana de Ciencias Aplicadas: Lima; 2011,

19. Sánchez-Moreno F. El sistema nacional de salud en el Perú. Rev Peru Med Exp Salud Publica. 2014;31(4):747-53.

20. Castillo E, Vasquez M. El rigor metodológico en la investigación cualitativa. Colombia Medica. 2003,34(3):164-167. 